\title{
MULTIPLE POSITIVE SOLUTIONS FOR A CHOQUARD EQUATION INVOLVING BOTH CONCAVE-CONVEX AND HARDY-LITTLEWOOD-SOBOLEV CRITICAL EXPONENT
}

\author{
R. ECharghaOUi, M. Khiddi AND S. M. SBAi
}

Abstract. In this paper, we consider a Choquard equation involving both concave-convex and Hardy-Littlewood-Sobolev critical exponent. By using the $\mathscr{N}$ ehari manifold, fibering maps and the Lusternik-Schnirelman category, we prove that the problem has at least cat $(\Omega)+1$ distinct positive solutions.

Mathematics subject classification (2010): 35J25, 35J60, 35A15.

Keywords and phrases: Choquard equation, $\mathscr{N}$ ehari manifold, Concave and convex nonlinearities, Lusternik-Schnirelman category.

\section{REFERENCES}

[1] Claudianor O. Alves, Fashun Gao, Marco Squassina, and Minbo Yang, Singularly perturbed critical choquard equations, Journal of Differential Equations 263 (7): 3943-3988, 2017.

[2] Claudianor O. Alves And Minbo YAng, Multiplicity and concentration of solutions for a quasilinear choquard equation, Journal of Mathematical Physics 55 (6): 061502, 2014.

[3] C. O. Alves AND Y. H. Ding, Multiplicity of positive solutions to a $p$-laplacian equation involving critical nonlinearity, Journal of Mathematical Analysis and Applications 279 (2): 508-521, 2003.

[4] Antonio Ambrosetti, Haïm Brezis, and Giovanna Cerami, Combined effects of concave and convex nonlinearities in some elliptic problems, Journal of Functional Analysis 122 (2): 519-543, 1994.

[5] VIERI BENCI AND GIOVANNA CERAMI, The effect of the domain topology on the number of positive solutions of nonlinear elliptic problems, Archive for Rational Mechanics and Analysis 114 (1): 79-93, 1991.

[6] Samira Benmouloud, Rachid Echarghaoui, And Si Mohammed Sbaï, Multiplicity of positive solutions for a critical quasilinear elliptic system with concave and convex nonlinearities, Journal of Mathematical Analysis and Applications 396 (1): 375-385, 2012.

[7] HAÏM BRÉZIS AND ELLIOTT LIEB, A relation between pointwise convergence of functions and convergence of functionals, Proceedings of the American Mathematical Society 88 (3): 486-490, 1983.

[8] Fashun Gao And Minbo YAng, On nonlocal choquard equations with Hardy-Littlewood-Sobolev criticalexponents, Journal of Mathematical Analysis and Applications 448 (2): 1006-1041, 2017.

[9] Fashun GaO AND MinBo YANG, On the Brezis-Nirenberg type critical problem for nonlinear choquard equation, Sci China Math, doi:10.1007(/s):10.1007/s11425-016-9067-5, 2017.

[10] TSING-SAN HSU, Multiple positive solutions for a critical quasilinear elliptic system with concaveconvex nonlinearities, Nonlinear Analysis: Theory, Methods and Applications 71 (7): 2688-2698, 2009.

[11] QIN Li And ZuOdong YANG, Multiplicity of positive solutions for a $p-q$-Laplacian system with concaveand critical nonlinearities, Journal of Mathematical Analysis and Applications 423 (1): 660680, 2015.

[12] Elliott H. Lieb AND Michael Loss, Analysis, volume 14 of graduate studies in mathematics, American Mathematical Society, Providence, RI, 4, 2001. 
[13] Elliott H. Lieb ANd BARry Simon, The hartree-fock theory for coulomb systems, Communications in Mathematical Physics 53 (3): 185-194, 1977.

[14] Vitaly Moroz and Jean Van Schaftingen, A guide to the choquard equation, preprint arXiv:1606.02158, 2016.

[15] Vitaly Moroz and Jean Van Schaftingen, A guide to the choquard equation, Journal of Fixed Point Theory and Applications, 19 (1): 773-813, 2017.

[16] Solomon IsAakovich PeKaR, Untersuchungen uber die Elektronentheorie der Kristalle, Akademie-Verlag, 1954.

[17] Gabriella TARANTello, On nonhomogeneous elliptic equations involving critical sobolev exponent, In Annales de l'Institut Henri Poincare (C) Non Linear Analysis, vol. 9, pp. 281-304, Elsevier, 2016.

[18] M. WILlEM, Minimax theorems, progress in nonlinear differential equations and applications 24, 1996. 